PAPER

\section{A symmetric system of mixed Painlevé III-V equations and its integrable origin}

To cite this article: H Aratyn et al 2016 J. Phys. A: Math. Theor. 49045201

View the article online for updates and enhancements.
Related content

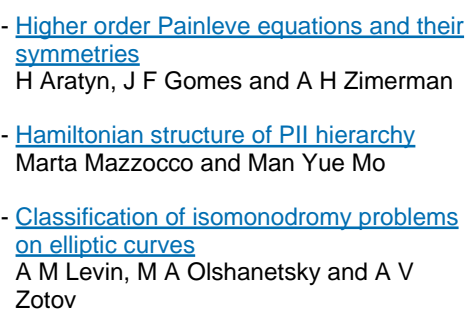

Recent citations

- Solutions of mixed Painlevé $\mathrm{P}_{\mathrm{H} \text { « }}$ model V C C Alves et al

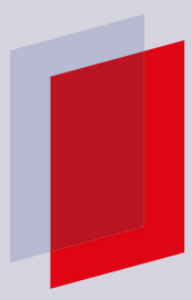

\section{IOP ebooks'}

Bringing you innovative digital publishing with leading voices to create your essential collection of books in STEM research. Start exploring the collection - download the first chapter of every title for free. 


\title{
A symmetric system of mixed Painlevé III-V equations and its integrable origin
}

\author{
H Aratyn ${ }^{1}$, J F Gomes ${ }^{2}$, D V Ruy ${ }^{2}$ and A H Zimerman ${ }^{2}$ \\ ${ }^{1}$ Department of Physics, University of Illinois at Chicago, 845 W. Taylor St., Chicago, \\ IL 60607, USA \\ ${ }^{2}$ Instituto de Física Teórica-UNESP Rua Dr Bento Teobaldo Ferraz 271, Bloco II \\ 01140-070, São Paulo, Brazil \\ E-mail: jfg@ift.unesp.br
}

Received 11 September 2015, revised 9 November 2015

Accepted for publication 16 November 2015

Published 18 December 2015

\begin{abstract}
A mixed symmetric Painlevé III-V model which describes a hybrid of both equations is defined and obtained by successive self-similarity and Dirac Lagrange multiplier reductions from an integrable 4-boson hierarchy.
\end{abstract}

Keywords: integrable hierarchies, Painlevé equations, nonlinear ordinary differential equations

\section{Introduction}

This paper deals with a system of nonlinear ordinary differential equations containing both Painlevé III and V equations as well as a new equation that passes the basic Painlevé test and possesses invariance under $A_{1}^{(1)}$ extended affine Weyl group. This system of equations emerges through self-similarity and Dirac reductions performed on a special class of integrable models $[3,5]$ referred to as multi-boson Lax hierarchies and presented here in a Hamiltonian setting.

As postulated by Ablowitz, Ramani and Segur (ARS) [2] the partial differential evolution equations of integrable hierarchies reduce in self-similarity limit to ordinary differential equations with solutions that have no movable critical points other than poles. This feature is known as Painlevé property.

The self-similarity reduction applied to multi-boson Lax hierarchies [3, 5] leads to higher Painlevé equations invariant under extended affine Weyl groups $A_{2 m}^{(1)}$ or $A_{2 m-1}^{(1)}, m=1,2 \ldots$ [14-17]. More specifically, the 4-boson model considered in this paper reduces in self-similarity limit to $A_{4}^{(1)}$ Painlevé equations. In another well-known example the 2-boson model reduces to $A_{2}^{(1)}$ Painlevé IV equations (see e.g. [6]). A construction given previously in [4-6] involved further reduction of $A_{4}^{(1)}$ Painlevé equation to $A_{3}^{(1)}$ Painlevé V (PV) equation. 
In this paper we are able to address the following question. How to reduce integrable models of $2 m$-boson type to Painlevé equations with symmetry structures other than $A_{2 m}^{(1)}$ or $A_{2 m-1}^{(1)}\left(m=2\right.$ in this paper)? For instance the Painlevé III equation with $B_{2}^{(1)}$ extended affine Weyl symmetry is here obtained as a limit of Dirac reduction of the $A_{4}^{(1)}$ Hamiltonian system. The two-step reduction of the 4-boson model, first to $A_{4}^{(1)}$ equations by the selfsimilarity reduction then followed by the Dirac reduction involving Lagrange multipliers leads to a new system of mixed symmetric Painlevé III-V equations that embed $A_{3}^{(1)} \mathrm{PV}$ equation and $B_{2}^{(1)}$ Painlevé III equation. Explicitly this new system is governed by the following equations:

$$
\begin{aligned}
z f_{i, z}= & f_{i} f_{i+2}\left(f_{i+1}-f_{i+3}\right)+(-1)^{i} f_{i}\left(\alpha_{1}+\alpha_{3}+C\right) \\
& +\alpha_{i}\left(f_{i}+f_{i+2}\right)-(-1)^{[i / 2]} \epsilon_{i+1}\left(f_{i+1}+f_{i+3}\right), \quad i=0,1,2,3,
\end{aligned}
$$

where $f_{i}=f_{i+4}$ and the symbol $[i / 2]$ is $i / 2$, if $i$ is even or $(i+1) / 2$, if $i$ is odd. Equation (1.1) have two features that distinguish them from standard symmetric $A_{3}^{(1)}$ PV equations [13-15]. First, one notices presence of additional terms that contain 'deformation parameters' $\epsilon_{i}$. These parameters satisfy periodicity conditions $\epsilon_{i}=\epsilon_{i+2}$ that reduce their number to two: $\epsilon_{0}$ and $\epsilon_{1}$. Secondly, in addition to usual parameters $\alpha_{i}, i=0,1$, 2, 3 of the affine Weyl structure the model also depends explicitly on an arbitrary parameter $C$.

Equation (1.1) possess two integration constants $r_{0}, r_{1}$ appearing in relations:

$$
f_{1}+f_{3}=r_{1} z^{-C}, \quad f_{0}+f_{2}=r_{0} z^{(C+\Omega)},
$$

where

$$
\Omega=\alpha_{0}+\alpha_{1}+\alpha_{2}+\alpha_{3} \neq 0 .
$$

For two special values of $C$ : $C=0$ or $C=-\Omega$ the combinations $f_{1}+f_{3}$ or $f_{0}+f_{2}$ become equal to integration constants $r_{1}$ or $r_{0}$, respectively.

For $C$ satisfying the condition

$$
C=-\Omega / 2 \text {. }
$$

the terms $f_{1}+f_{3}$ and $f_{0}+f_{2}$ are proportional to each other by a constant. Such condition was assumed in [13-15]. When the condition (1.4) holds equation (1.1) describes either Painlevé III or PV equation. More explicitly, equation (1.1) will turn into Painlevé III or PV equations depending on whether the integration constant $r_{1}$ vanishes or has a non-zero value (to simplify the argument we assumed here that constants $r_{0}$ and $\epsilon_{0}$ remain different from zero). Thus in the case of (1.4) (and $r_{0} \neq 0$ ) equation (1.1) has effectively only one deformation parameter $r_{1}$ determining transition between Painlevé III and $\mathrm{V}$ cases.

The symmetry structure of equation (1.1) possesses additional features when $C$ fails to satisfy the condition (1.4), which we illustrate here by considering $C=0$. In this case (and with $r_{0}=1, \epsilon_{1}=0$ ) this model is described by a second order differential equation:

$$
\begin{aligned}
q_{z z}= & -\frac{1}{z} q_{z}+\left(\frac{1}{2 q}+\frac{1}{2\left(q-r_{1}\right)}\right)\left(q_{z}^{2}-\epsilon_{0}^{2}\right)-\frac{\left(\alpha r_{1}-\epsilon_{0} z \alpha_{3}\right) q}{z^{2}\left(q-r_{1}\right)} \\
& -\frac{\beta r_{1}\left(q-r_{1}\right)+\epsilon_{0} r_{1} z \alpha_{1}}{z^{2} q}-\frac{\gamma q\left(q-r_{1}\right)-\epsilon_{0}\left(1+\alpha_{1}\right)}{z} \\
& +\frac{1}{2} q\left(q-r_{1}\right)\left(2 q-r_{1}\right),
\end{aligned}
$$


where $q=f_{1}$ and with $\alpha, \beta, \gamma$ parameters to be defined below in relation (4.35). The other two parameters present in the above equation are: a deformation parameter $\epsilon_{0}$ and an integration constant $r_{1}$.

For non-zero values of these two parameters equation (1.5) describes a system which is neither Painlevé III nor V equation but a new equation with only $A_{1}^{(1)}$ symmetry. It will be shown that equation (1.5) passes the Painlevé test for all values of its parameters. Thus our study indicates that Dirac reduction of Hamiltonian systems preserves the Painlevé property. By letting one of these parameters $\epsilon_{0}$ or $r_{1}$ go to zero one recovers PV or Painlevé III equations. More specifically, in the limit $\epsilon_{0} \rightarrow 0$ the equation (1.5) becomes the PV equation with $A_{3}^{(1)}$ extended affine Weyl symmetry after transformation from $q$ to $y=1-r_{1} / q$. On the other hand, in the limit $r_{1} \rightarrow 0$ the equation (1.5) goes into the Painlevé III equation invariant under $B_{2}^{(1)}$ extended affine Weyl symmetry.

The schematic diagram of our presentation is shown below:

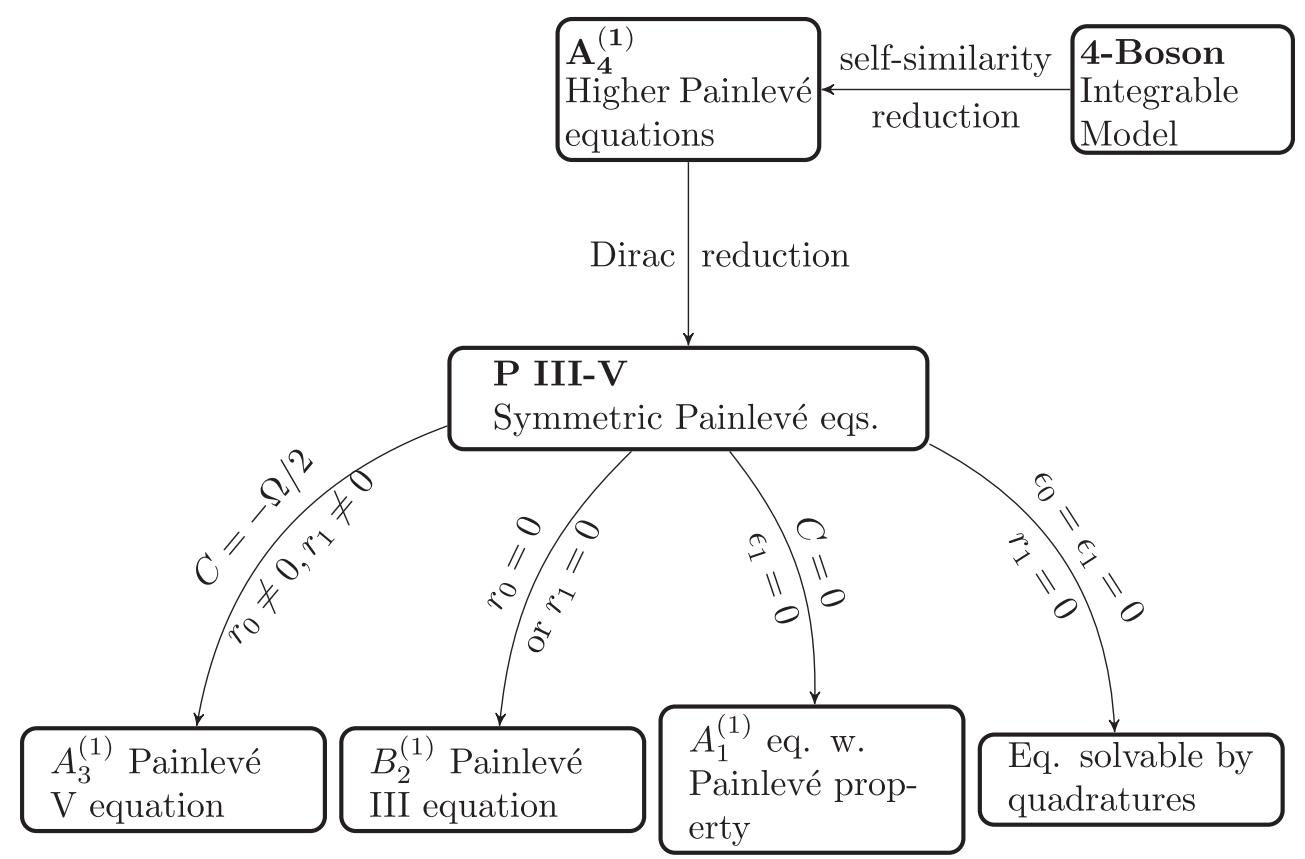

The above diagram summarizes main results of our construction. Each of the Painlevé equations listed at the bottom of the diagram and associated with $A_{3}^{(1)}, B_{2}^{(1)}, A_{1}^{(1)}$ symmetry structures can be identified with reductions of higher Painlevé equations of $A_{4}^{(1)}$ type via the Dirac Lagrange multiplier method within the Hamiltonian framework. All these equations can be embedded in a new set of symmetric symmetric Painlevé III-V equations and then recovered by taking appropriate limits of the underlying parameters $C, r_{i}, \epsilon_{i}$, $i=1,2$.

In section 2 we obtain higher Painlevé equations in a self-similarity limit of the 4-boson integrable model and recall their invariance under the extended affine Weyl $A_{4}^{(1)}$ group of Bäcklund transformations.

In section 3 we work within the Hamiltonian formalism applying Dirac Lagrange multiplier method [10] on $A_{4}^{(1)}$ model to obtain Hamiltonian structures governing symmetric Painlevé III-V model. 
In section 4 we discuss symmetric Painlevé III-V equations and their reductions. In section 4.1 we introduce a version of symmetric PV equations with explicit dependence on an arbitrary constant $C$ that determines form of the Bäcklund automorphism $\pi$ of the extended affine $A_{3}^{(1)}$ Weyl group. By adding additional terms to symmetric PV equations that break ambiguity in the value of $C$ we arrive at the notion of symmetric Painlevé III-V equations presented in section 4.2. Various submodels obtained by setting the underlying constants to specific values and corresponding to symmetry structures, $A_{3}^{(1)}, B_{2}^{(1)}, A_{1}^{(1)}$, as well as a solvable model, are discussed in separate subsections of this section. First, in 4.2 we point out conditions, $C=-\Omega / 2, r_{0} \neq 0, r_{1} \neq 0$, on $C, r_{1}, r_{2}$ under which symmetric Painlevé III-V equations reduce to PV equations. In section 4.3 we reduce symmetric Painlevé III-V equations to symmetric Painlevé III equations in the $r_{1} \rightarrow 0$ limit for any $C$ and study emergence of $B_{2}^{(1)}$ symmetry group out of $A_{3}^{(1)}$ in this limit. Hamiltonian representations of symmetric Painlevé III-V equations are introduced in section 4.4. In section 4.4.1 it shown how for $C=-\Omega / 2$ all Hamiltonian equations can be derived from one model with the fixed value of $C$ taken here to be equal to $-1 / 2$. Thus, the Dirac reduction of section 3 carried out for $C=-1 / 2$ (and $C=0$ ) can be considered established for all values of $C=-\Omega / 2$. In sections 4.4.2 and 4.4.3 we extend the Hamiltonian representations to cases of $C \neq-\Omega / 2$. Especially in section 4.4.3 we cover the case of $C=0$ and perform the Painlevé analysis of $A_{1}^{(1)}$ equation (1.5) obtained for $C=0, r_{0}=1$ and $\epsilon_{1}=0$ that is a mixture of Painlevé III and $\mathrm{V}$ equations.

In a final section 5 we offer concluding remarks and discuss planned extensions of this work.

\section{Self-similarity reduction of 4-boson integrable model to $A_{4}^{(1)}$ Painlevé system}

The integrable 4-boson model is defined by the pseudo-differential Lax operator:

$$
\begin{aligned}
L= & \left(\frac{\partial}{\partial x}-e_{2}\right)\left(\frac{\partial}{\partial x}-e_{1}-c_{2}\right)\left(\frac{\partial}{\partial x}-c_{1}-c_{2}\right) \\
& \times\left(\frac{\partial}{\partial x}-e_{1}-c_{1}-c_{2}\right)^{-1}\left(\frac{\partial}{\partial x}-e_{2}-c_{2}\right)^{-1},
\end{aligned}
$$

and is a particular case of the $2 m$-boson sub-hierarchy of the KP hierarchy with Lax operators that are given by a ratio of products:

$$
L_{m}=\prod_{j=1}^{m+1}\left(\frac{\partial}{\partial x}+v_{m+2-j}\right) \prod_{l=1}^{m}\left(\frac{\partial}{\partial x}+\tilde{v}_{l}\right)^{-1}, \quad m=1,2, \ldots
$$

The Lax coefficients of this sub-hierarchy are subject to the constraint:

$$
\sum_{j=1}^{m+1} v_{j}-\sum_{l=1}^{m} \tilde{v}_{l}=0
$$

and the underlying second bracket structure has a form of graded $S L(m+1, m)$ Kac-Moody algebra in a diagonal gauge. As shown in $[3,4]$ the second bracket structure is diagonalized by a change of variables to $m$ conjugated pairs $\left(c_{i}, e_{i}\right)_{i=1, \ldots, m}$ through relations

$$
\tilde{v}_{l}=-e_{l}-\sum_{p=l}^{m} c_{p}, \quad v_{j}=-e_{j-1}-\sum_{p=j}^{m} c_{p}
$$


for $l=1,2, \ldots, m$ and $j=1, \ldots, m+1$. These relations automatically solve the constraint and re-produce the graded $S L(m+1, m)$ bracket structure from a simple bracket structure

$$
\left\{e_{i}(x,[t]), c_{j}(y,[t])\right\}_{2}=-\delta_{i j} \delta_{x}(x-y), i, j=1,2, \ldots, m,
$$

where coefficients are taken at equal higher times $[t]=t_{2}, t_{3}, \ldots[3,4]$.

The $2 m$-boson sub-hierarchy is characterized by invariance under Darboux-Bäcklund symmetry transformations associated with a special Volterra lattice symmetry structure within the bigger structure of Toda lattice [3].

The system of partial differential equations describing the second $t_{2}$ - flow of the 4-boson integrable hierarchy [3]:

$$
\begin{aligned}
& \frac{\partial c_{1}}{\partial t_{2}}=\frac{\partial}{\partial x}\left(c_{1, x}-c_{1}^{2}-2 e_{1} c_{1}+2 c_{2, x}-2 c_{1} c_{2}\right) \\
& \frac{\partial e_{1}}{\partial t_{2}}=\frac{\partial}{\partial x}\left(-e_{1, x}-e_{1}^{2}-2 e_{1} c_{1}-2 e_{1} c_{2}\right) \\
& \frac{\partial c_{2}}{\partial t_{2}}=\frac{\partial}{\partial x}\left(c_{2, x}-c_{2}^{2}-2 e_{2} c_{2}\right) \\
& \frac{\partial e_{2}}{\partial t_{2}}=\frac{\partial}{\partial x}\left(-e_{2, x}-e_{2}^{2}-2 e_{2} c_{2}-2\left(e_{1, x}+c_{1} e_{1}\right)\right)
\end{aligned}
$$

reduces in a self-similarity limit to ordinary differential equations with a Painlevé property, in agreement with the ARS conjecture [2]. The Painlevé equations obtained from equations (2.3) in such limit will be a subject of this section.

To perform self-similarity reduction we introduce a variable $\xi=x / \sqrt{t_{2}}$ and define $\tilde{e}_{j}(\xi)$ and $\tilde{c}_{j}(\xi)$ through relations

$$
\tilde{c}_{j}(\xi)=c_{j}\left(x, t_{2}\right) \sqrt{t_{2}}, \tilde{e}_{j}(\xi)=e_{j}\left(x, t_{2}\right) \sqrt{t_{2}}, \quad j=1,2 .
$$

Equations (2.3) in a self-similarity limit simplify when rewritten in terms of

$Y_{1}=\tilde{e}_{1}+\tilde{c}_{1}+2 \tilde{c}_{2}, Y_{2}=\tilde{e}_{2}+\tilde{c}_{2}$ and $\tilde{e}_{i}(\xi), i=1,2$, that in this limit form two pairs of conjugated canonical variables. To further streamline notation we will drop the tilde over $\tilde{e}_{i}(\xi)$. In this notation a self-similarity limit of equation (2.3) takes a form of Hamilton equations:

$$
e_{j, \xi}=\frac{\partial \mathcal{H}_{A_{4}^{(1)}}}{\partial Y_{j}}, Y_{j, \xi}=-\frac{\partial \mathcal{H}_{A_{4}^{(1)}}}{\partial e_{j}}, j=1,2 .
$$

obtained from the Hamiltonian

$$
\begin{aligned}
\mathcal{H}_{A_{4}^{(1)}}\left(e_{1}, Y_{1}, e_{2}, Y_{2}\right)= & -\sum_{j=1}^{2} e_{j}\left(Y_{j}-\frac{\xi}{2}\right)\left(Y_{j}-e_{j}\right)+2 e_{1}\left(Y_{1}-\frac{\xi}{2}\right)\left(Y_{2}-e_{2}\right) \\
& +\bar{k}_{1} Y_{1}+\bar{k}_{2} Y_{2}-k_{1} e_{1}-k_{2} e_{2}
\end{aligned}
$$

with arbitrary constants $k_{i}, \bar{k}_{i}, i=1,2$. The Hamilton equations are consistent with the following bracket structure

$$
\left\{e_{i}, Y_{j}\right\}=\delta_{i j}, \quad i, j=1,2 .
$$


The Hamiltonian (2.5) can be rewritten as [5]:

$\mathcal{H}_{A^{(1)} 4}=\sum_{j=1}^{2} p_{j} q_{j}\left(p_{j}+q_{j}+\xi / 2\right)+2 p_{1} q_{1} p_{2}-\sum_{j=1}^{2} \alpha_{2 j} q_{j}+\sum_{j=1}^{2} p_{j}\left(\sum_{k=1}^{j} \alpha_{2 k-1}\right)$

by employing a symplectic transformation:

$$
\begin{array}{ll}
e_{2}=p_{2}+q_{2}+\xi / 2, & e_{1}=-q_{1} \\
Y_{2}=p_{2}+q_{1}+\xi / 2, & Y_{1}=-q_{2}-p_{2}-p_{1}
\end{array}
$$

and setting:

$$
\alpha_{1}=-\bar{k}_{1}, \quad \alpha_{2}=-k_{1}-\bar{k}_{2}, \quad \alpha_{3}=\bar{k}_{2}-k_{2}, \quad \alpha_{4}=k_{2}+\bar{k}_{1} .
$$

We now can identify a self-similarity limit of (2.3) with $A_{4}^{(1)}$ Painlevé equations:

$$
f_{i, \xi}=f_{i}\left(f_{i+1}-f_{i+2}+f_{i+3}-f_{i+4}\right)+\alpha_{i}, \quad i=0, \ldots, 4
$$

realized from a polynomial Hamiltonian (2.7) through relations

$$
p_{i}=f_{2 i}, \quad q_{i}=\sum_{k=1}^{i} f_{2 k-1}, \quad i=1, \ldots, 4,
$$

when conditions $f_{0}+\cdots+f_{4}=-\xi / 2$ and $\alpha_{0}+\cdots+\alpha_{4}=-1 / 2$ are satisfied.

The above Painlevé equation (2.9) are manifestly invariant under the following Bäcklund transformations:

$s_{i}\left(\alpha_{i}\right)=-\alpha_{i}, \quad s_{i}\left(\alpha_{j}\right)=\alpha_{j}+\alpha_{i}(j=i \pm 1), s_{i}\left(\alpha_{j}\right)=\alpha_{j}(j \neq i, i \pm 1)$,

$s_{i}\left(f_{i}\right)=f_{i}, \quad s_{i}\left(f_{j}\right)=f_{j} \pm \frac{\alpha_{i}}{f_{i}}(j=i \pm 1), s_{i}\left(f_{j}\right)=f_{j} \quad(j \neq i, i \pm 1)$,

$\pi\left(\alpha_{j}\right)=\alpha_{j+1}, \quad \pi\left(f_{j}\right)=f_{j+1}, \quad i, j=0,1, \ldots, 4$

of the extended affine Weyl group $A_{4}^{(1)}$. The generators $\pi, s_{i}, i=0,1, \ldots, 4$ satisfy the following fundamental relations $[1,14-17]$ :

$s_{i}^{2}=1, \quad s_{i} s_{j}=s_{j} s_{i}(j \neq i, i \pm 1), s_{i} s_{j} s_{i}=s_{j} s_{i} s_{j}(j=i \pm 1)$,

$\pi^{4}=1, \quad \pi s_{j}=s_{j+1} \pi$

\section{Dirac Lagrange multiplier reduction of $\mathcal{H}_{A_{4}^{(1)}}$}

Our starting point is the Hamiltonian $\mathcal{H}_{A_{4}^{(1)}}(2.5)$ describing a self-similarity reduction of the 4-boson model.

We first define a pair of canonical variables $P, Q$ :

$$
P=-N \xi e_{1}, \quad Q=\frac{Y_{1}-M \xi}{N \xi}
$$

via symplectic transformation from $e_{1}, Y_{1}$ involving constants $M, N$ and a variable $\xi$ from previous section. This change of variables redefines the corresponding Hamiltonian $\mathcal{H}_{A_{4}^{(1)}}$ as follows:

$$
\mathcal{H}_{A_{4}^{(1)}}\left(P, Q, e_{2}, Y_{2}\right)=\mathcal{H}_{A_{4}^{(1)}}\left(e_{1}, Y_{1}, e_{2}, Y_{2}\right)-\frac{P Q}{\xi}-\frac{M P}{N \xi}
$$


so that $\mathcal{H}_{A_{4}^{(1)}}$ in terms of $e_{2}, Y_{2}, P, Q$ becomes:

$$
\begin{aligned}
& \mathcal{H}_{A_{4}^{(1)}}\left(P, Q, e_{2}, Y_{2}\right) \\
&=-\frac{1}{\left(2 \xi N^{2}\right)}\left(-2 M P^{2}+P^{2}-2 \bar{k}_{2} Y_{2} N^{2} \xi-2 k_{1} P N\right. \\
& \quad+2 k_{2} e_{2} N^{2} \xi+2 P Q N^{2}+2 M P N-2 P Q^{2} N^{3} \xi^{2} \\
& \quad-2 P M^{2} \xi^{2} N+P Q N^{2} \xi^{2}+P M \xi^{2} N-2 Q N P^{2} \\
&+e_{2}^{2} N^{2} \xi^{2}-4 P Q N^{2} M \xi^{2}+2 e_{2} N^{2} \xi Y_{2}^{2}-2 e_{2}^{2} N^{2} \xi Y_{2} \\
&-e_{2} N^{2} \xi^{2} Y_{2}-2 P \xi N Y_{2}+2 P \xi N e_{2}-2 \bar{k}_{1} \xi^{2} N^{3} Q \\
&\left.+4 P \xi N^{2} Q Y_{2}-4 P \xi N^{2} Q e_{2}+4 P \xi N M Y_{2}-4 P \xi N M e_{2}\right)
\end{aligned}
$$

up to constant terms.

To eliminate variables $e_{2}$ and $Y_{2}$ we impose two second class constraints:

$$
\begin{aligned}
& \phi_{1}=e_{2}-\frac{1}{\xi}\left(D \xi^{2}+E P+F P Q\right), \\
& \phi_{2}=Y_{2}-A \xi
\end{aligned}
$$

with constants $D, E, F, A$ (together with $N, M$ introduced earlier) to be completely determined by the condition that the reduction process reproduces one of the following Hamiltonian systems:

- The Hamiltonian system that will be shown to describe equation (1.1) for $C=0, r_{0}=1$, $\Omega=1$ and $\epsilon_{1}=0$ and $q=f_{1}, p=-f_{2}$ as in relation (4.24):

$$
z H_{C=0}=p(p+z) q\left(q-r_{1}\right)+\alpha_{2} z q+\left(\alpha_{1} r_{1}+\epsilon_{0} z\right) p-\left(\alpha_{1}+\alpha_{3}\right) p q .
$$

- The Hamiltonian system that will be shown to describe equation (1.1) for $C=-1 / 2$, $\Omega=1, r_{0}=1$ and $\epsilon_{1}=-1$ and for $q=f_{1} z^{-1 / 2}, p=-f_{2} z^{1 / 2}$ as in relation (4.27):

$$
\begin{aligned}
z H_{C=-1 / 2}= & p(p+z) q\left(q-r_{1}\right)-\left(\alpha_{1}+\alpha_{3}\right) p q+\left(\alpha_{1} r_{1}+\epsilon_{0}\right) p \\
& +\left(\alpha_{2}-r_{1}\right) z q .
\end{aligned}
$$

Here we define $z$ as $z=\xi^{2}$.

Below in section 4.4 we will discuss how these two Hamiltonian structures fit into the formalism of symmetric PIII-V equations and show that all the Hamilton equations with $C=-\Omega / 2$ can be obtained from $H_{C=-1 / 2}$ by simple rescaling.

We will now follow the Lagrange multiplier approach to impose the constraints from (3.2) by augmenting the Hamiltonian $\mathcal{H}_{A_{4}^{(1)}}$ by Lagrange multiplier terms:

$$
\mathcal{H}_{A_{4}^{(1)}} \rightarrow \mathcal{H}_{A_{4}^{(1)}}^{\lambda}=\mathcal{H}_{A_{4}^{(1)}}+\lambda_{1} \phi_{1}+\lambda_{2} \phi_{2}
$$

The Lagrange multipliers $\lambda_{1}, \lambda_{2}$ are fixed by condition of compatibility of constraint $\phi_{i}, i=1$, 2:

$$
0=\frac{\mathrm{d} \phi_{m}}{\mathrm{~d} \xi}=\left\{\phi_{m}, H_{A_{4}{ }^{(1)}}\right\}+\frac{\partial \phi_{m}}{\partial \xi}+\sum_{n=1}^{2} \lambda_{n}\left\{\phi_{m}, \phi_{n}\right\}
$$

on the constraint manifold. These compatibility conditions fix the values of Lagrange multipliers to: 


$$
\lambda_{n}=-\sum_{n=1}^{2}\left\{\phi_{n}, \phi_{m}\right\}^{-1}\left(\left\{\phi_{m}, H_{A_{4}^{(1)}}\right\}+\frac{\partial \phi_{m}}{\partial \xi}\right)
$$

where the values of the matrix elements of the inverse of the matrix $\left\{\phi_{n}, \phi_{m}\right\}$ are $\left\{\phi_{1}, \phi_{2}\right\}^{-1}=-1=-\left\{\phi_{2}, \phi_{1}\right\}^{-1}$ and zero otherwise. Thus

$$
\mathcal{H}_{A_{4}^{(1)}}^{\lambda}=\mathcal{H}_{A_{4}^{(1)}}-\sum_{n=1, m=1}^{2} \phi_{n}\left\{\phi_{n}, \phi_{m}\right\}^{-1}\left(\left\{\phi_{m}, H_{A_{4}^{(1)}}\right\}+\frac{\partial \phi_{m}}{\partial \xi}\right)
$$

and equations of motion for $P, Q$ become

$$
\begin{aligned}
& P_{\xi}=\left\{P, H_{A_{4}^{(1)}}\right\}-\left.\left\{P, \phi_{1}\right\}\left\{\phi_{1}, \phi_{2}\right\}^{-1}\left(\left\{\phi_{2}, H_{A_{4}^{(1)}}\right\}+\frac{\partial \phi_{2}}{\partial \xi}\right)\right|_{\phi_{i} \approx 0} \\
& Q_{\xi}=\left\{Q, H_{A_{4}^{(1)}}\right\}-\left.\left\{Q, \phi_{1}\right\}\left\{\phi_{1}, \phi_{2}\right\}^{-1}\left(\left\{\phi_{2}, H_{A_{4}^{(1)}}\right\}+\frac{\partial \phi_{2}}{\partial \xi}\right)\right|_{\phi_{i} \approx 0}
\end{aligned}
$$

Explicit calculation gives the following values for quantities appearing in (3.7):

$\left\{\phi_{2}, H_{A_{4}^{(1)}}\right\}=-\frac{\partial H_{A_{4}^{(1)}}}{\partial e_{2}}, \quad\left\{P, \phi_{1}\right\}=\frac{1}{\xi} F P, \quad\left\{Q, \phi_{1}\right\}=-\frac{1}{\xi}(E+F Q)$.

Multiplying both sides of equations (3.7) by $\xi$ in order to obtain expressions for $\xi P_{\xi}, \xi Q_{\xi}$ and inserting the technical results (3.8) yields:

$$
\begin{aligned}
& \xi P_{\xi}=-\xi \frac{\partial H_{A_{4}^{(1)}}}{\partial Q}-\left.F P\left(\frac{\partial H_{A_{4}^{(1)}}}{\partial e_{2}}+A\right)\right|_{\phi_{i} \approx 0} \\
& \xi Q_{\xi}=\xi \frac{\partial H_{A_{4}^{(1)}}}{\partial P}+\left.(E+F Q)\left(\frac{\partial H_{A_{4}^{(1)}}}{\partial e_{2}}+A\right)\right|_{\phi_{i} \approx 0}
\end{aligned}
$$

We will first compare equations (3.9) to the Hamilton equations:

$$
\begin{aligned}
& z q_{z}=q\left(q-r_{1}\right)(2 p+z)-\left(\alpha_{1}+\alpha_{3}\right) q+\alpha_{1} r_{1}+\epsilon_{0} z \\
& z p_{z}=-p(p+z)\left(2 q-r_{1}\right)+\left(\alpha_{1}+\alpha_{3}\right) p-\alpha_{2} z
\end{aligned}
$$

obtained from $H_{C}=0$ given in (3.3). For $z=\xi^{2}$ these equations can be rewritten as

$$
\xi q_{\xi}=2 \frac{\partial H_{C=0}}{\partial p}, \quad \xi p_{\xi}=-2 \frac{\partial H_{C=0}}{\partial q} .
$$

Equations (3.9a) and (3.9b) will agree with equation (3.11) when we identify $P=p$ and $Q=q$ and values of $N, M, D, E, F, A$ become functions of $r_{1}$ and $\epsilon_{0}$ that will now be given below.

We find that

$$
N=2, \quad A=\frac{1}{2}+\frac{32 \epsilon_{0}}{16 r_{1}^{2}-1}
$$

and $F \neq 0$ is a solution of a quadratic equation:

$$
16 \epsilon_{0} F^{2}+\left(16 r_{1}^{2}-1\right)(F-1)=0 .
$$

Note, that for $16 r_{1}^{2}-1=0$ or $r_{1}= \pm 1 / 4$ it must hold that $\epsilon_{0}=0$. It is convenient to express the remaining parameters $M, D, E$ in terms of $F$ being a solution (3.12) and an auxiliary quantity: 


$$
G=1-F+4 r_{1}^{2} F^{2}=F^{2} \frac{\epsilon_{0}^{2}+r_{1}^{2} / 4-4 r_{1}^{4}}{1 / 16-r_{1}^{2}}
$$

as

$$
\begin{aligned}
& D=\frac{1}{F^{2}}\left(-F+F^{2} / 4+1 \pm \frac{1}{2} \sqrt{G}\right), \\
& =\frac{1}{2}\left(\frac{1}{2}+\frac{32 \epsilon_{0}}{16 r_{1}^{2}-1}\right) \pm \frac{1}{2} \sqrt{\frac{\epsilon_{0}^{2}+r_{1}^{2} / 4-4 r_{1}^{4}}{1 / 16-r_{1}^{2}}}, \\
& M=\frac{1}{F^{3}}\left(\left(1-2 r_{1}\right) F^{3}-2 F^{2}+2 F \pm F(F-2) \sqrt{G}\right), \\
& E=-\frac{1}{4 F}\left(2 r_{1} F^{2}+F \pm F \sqrt{G}\right) .
\end{aligned}
$$

In addition the following conditions on constants $k_{1}, k_{2}, \bar{k}_{1}$ need to hold:

$$
\begin{aligned}
\bar{k}_{1}= & \alpha_{2}, \\
k_{2}= & \frac{1}{2 F^{2}}\left(F^{2}+4-6 F+4 F\left(\alpha_{1}+\alpha_{3}\right)\right), \\
k_{1}= & \frac{1}{2 F^{3}}\left(F^{3}\left(1+4 r_{1}\left(\alpha_{1}-\alpha_{3}\right)\right)+2 F-F^{2}\left(2\left(\alpha_{1}+\alpha_{3}\right)+1\right)+\right. \\
& \left. \pm 2 F\left(F\left(\alpha_{1}+\alpha_{3}\right)-1\right) \sqrt{G}\right) .
\end{aligned}
$$

Next we determine conditions for which equations (3.9) and the Hamilton equations

$$
\begin{aligned}
& z q_{z}=q\left(q-r_{1}\right)(2 p+z)-\left(\alpha_{1}+\alpha_{3}\right) q+\alpha_{1} r_{1}+\epsilon_{0} \\
& z p_{z}=-p(p+z)\left(2 q-r_{1}\right)+\left(\alpha_{1}+\alpha_{3}\right) p-\alpha_{2} z+r_{1} z
\end{aligned}
$$

will agree. Equation (3.21) are obtained from the Hamilton $H_{C=-1 / 2}$ (see definition (3.4)), which as will be shown in the next section describes (1.1) for a special non-zero value of the parameter $C(C=-1 / 2)$ and $r_{0}=1, \epsilon_{1}=-1$. Since we identify $z=\xi^{2}$ the comparison is to be made with equations

$$
\xi q_{\xi}=2 \frac{\partial H_{C=-1 / 2}}{\partial p}, \quad \xi p_{\xi}=-2 \frac{\partial H_{C=-1 / 2}}{\partial q},
$$

This procedure fixes the values of $N, M, D, E, F, A$ to

$$
F=1, \quad A=\frac{1}{2}, \quad N=2, \quad D=\frac{1}{4} \pm r_{1}
$$

and

$$
E=\left\{\begin{array}{l}
-\frac{1}{4} \\
-\left(r_{1}+1 / 4\right)
\end{array}, \quad M=\left\{\begin{array}{l}
1 / 2-2 r_{1} \\
1 / 2
\end{array}\right.\right.
$$

and

$$
\bar{k}_{1}=\alpha_{2}-r_{1},
$$




$$
\begin{aligned}
& k_{2}=\frac{3}{2}-2\left(\alpha_{1}+\alpha_{3}\right) \\
& k_{1 \pm}=\alpha_{0}+\alpha_{2}+2 r_{1}\left(2 \alpha_{1}-1\right)+4 \epsilon_{0}+\left\{\begin{array}{l}
0 \\
4\left(\alpha_{0}+\alpha_{2}\right) r_{1}
\end{array}\right.
\end{aligned}
$$

In conclusion, we have obtained two Hamilton equations (3.10) and (3.21) by Dirac reduction of the self-similarity limit of the 4-boson model. In the next section the Hamilton equations (3.10) and (3.21) obtained in this section will be identified with generic examples of new symmetric PIII-PV equations.

\section{Symmetric formulation of Painlevé III and V systems}

\subsection{On symmetric $A_{3}^{(1)}$ Painlevé equations}

To provide a useful framework for combining reductions of systems described in the previous section we first recall symmetric $A_{3}^{(1)} \mathrm{PV}$ equations (see e.g. [14]):

$$
\begin{aligned}
& z f_{i, z}=f_{i} f_{i+2}\left(f_{i+1}-f_{i+3}\right)+(-1)^{i} f_{i}\left(\alpha_{1}+\alpha_{3}+C\right) \\
& +\alpha_{i}\left(f_{i}+f_{i+2}\right), \quad i=0,1,2,3,
\end{aligned}
$$

where $C$ and $\alpha_{i}, i=0,1,2,3$ are being constants. Unlike the [14] we do not assume relation (1.4) between $C$ and $\Omega$.

Equation (4.1) are manifestly invariant under the extended affine Weyl group $A_{3}^{(1)}$ generators $\pi, s_{i}, i=0,1,2,3$ acting as defined in relations (2.11). However the action of the automorphism operator $\pi$ depends on whether a parameter $C$ satisfies the condition (1.4) as we will now discuss.

By summing $i=0,2$ and $i=1,3$ components of equation (4.1) one obtains

$$
\begin{aligned}
& z \frac{\mathrm{d}\left(f_{0}+f_{2}\right)}{\mathrm{d} z}=\left(f_{0}+f_{2}\right)\left(C+\alpha_{0}+\alpha_{1}+\alpha_{2}+\alpha_{3}\right), \\
& z \frac{\mathrm{d}\left(f_{1}+f_{3}\right)}{\mathrm{d} z}=\left(f_{1}+f_{3}\right)(-C),
\end{aligned}
$$

which can be rewritten as equation (1.2), revealing two integration constants $r_{0}$ and $r_{1}$ of equation (4.1). Note that having identical equation $z\left(f_{i}+f_{i+2}\right)_{z}=(-C)\left(f_{i}+f_{i+2}\right)$ for both $i=0$ and $i=1$ is a necessary condition for canonical $\left(\pi\left(f_{i}\right)=f_{i+1}\right)$ transformation rule for $\pi$. This requires the condition (1.4) with $r_{0}=r_{1}$. When the condition (1.4) holds one can cast equation (1.2) in a compact form;

$$
f_{i}+f_{i+2}=r_{i} z^{-C}, \quad i=0,1
$$

We also observe that the substitution:

$$
\bar{f}_{j}=z^{\kappa} f_{j}, \quad j=1,3 \quad \bar{f}_{i}=f_{i} / z^{\kappa}, \quad i=0,2
$$

in equation (4.1) shifts the value of $C$ to $\bar{C}=C-\kappa$ and changes the canonical action of $\pi$ automorphism to:

$$
\pi\left(\bar{f}_{2 j}\right)=\frac{1}{z^{2 \kappa}} \bar{f}_{2 j+1}, \quad \pi\left(\bar{f}_{2 j-1}\right)=z^{2 \kappa} \bar{f}_{2 j} .
$$


Despite its non-conventional form such operator $\pi$ continues to satisfy the extended affine Weyl group fundamental relations (2.12). In particular, for the choice $\kappa=C$ the value of $\bar{C}=C-\kappa$ becomes equal to zero. One should add that setting explicitly $C=0$ in equation (4.1) with $\Omega=1, r_{0}=1$ as it was done in [20] results in invariance under $\pi$ given by

$$
\pi\left(\alpha_{i}\right)=\alpha_{i+1}, \quad \pi\left(f_{2 i}\right)=\frac{z}{r_{1}} f_{2 i+1}, \quad \pi\left(f_{2 i-1}\right)=\frac{r_{1}}{z} f_{2 i},
$$

which agrees with above discussion on consequences of shifting the value of $C$ in (4.1) for the form of automorphism $\pi$.

Thus for the symmetric Painlevé V equation (4.1) one is able to vary the value of the parameter $C$. The equation (4.1) remain invariant under the extended affine Weyl symmetry although such transformations of $C$ modify a form of the automorphism $\pi$.

\subsection{Symmetric PIII-PV equations}

In this subsection, we propose a model that combines Painlevé III and PV equations and study its properties.

We start our discussion by noticing an obvious ambiguity in the definition (4.1).

For a fixed value of $\alpha_{0}+\alpha_{1}+\alpha_{2}+\alpha_{3}=\Omega \neq 0$, let us consider adding additional terms $(-1)^{[i / 2]} \kappa_{i}\left(f_{i}+f_{i+2}\right)$ in (4.1):

$$
\begin{aligned}
z f_{i, z}= & f_{i} f_{i+2}\left(f_{i+1}-f_{i+3}\right)+(-1)^{i} f_{i}\left(\alpha_{1}+\alpha_{3}+C\right) \\
& +\alpha_{i}\left(f_{i}+f_{i+2}\right)-(-1)^{[i / 2]} \kappa_{i}\left(f_{i}+f_{i+2}\right),
\end{aligned}
$$

where $\kappa_{i}=\kappa_{i+2}$. Despite the presence of additional terms with $\kappa_{i}$ equation (4.6) remains invariant under the extended affine Weyl group $A_{3}^{(1)}$ with transformations (2.11) as long as one substitutes $\alpha_{i}$ by $\bar{\alpha}_{i}=\alpha_{i}-(-1)^{[i / 2]} \kappa_{i}$.

We have seen above that the parameter $C$ in (4.1) can be shifted by a simple re-scaling of $f_{i}$ 's that keeps (4.1) invariant but in the process changes the form of action of $\pi$.

In order to fix the value of the parameter $C$ we now define a system shown in (1.1), which for the convenience of the reader we reproduce here:

$$
\begin{aligned}
z f_{i, z}= & f_{i} f_{i+2}\left(f_{i+1}-f_{i+3}\right)+(-1)^{i} f_{i}\left(\alpha_{1}+\alpha_{3}+C\right) \\
& +\alpha_{i}\left(f_{i}+f_{i+2}\right)-(-1)^{[i / 2]} \epsilon_{i+1}\left(f_{i+1}+f_{i+3}\right), \quad i=0,1,2,3 .
\end{aligned}
$$

There are two deformation parameters involved in this construction; $\epsilon_{0}\left(=\epsilon_{2}\right)$ and $\epsilon_{1}\left(=\epsilon_{3}\right)$.

The system (1.1) is invariant under $\pi\left(\alpha_{j}\right)=\alpha_{j+1}, \pi\left(f_{j}\right)=f_{j+1}, \pi\left(\epsilon_{j}\right)=(-1)^{j+1} \epsilon_{j+1}$ but the equation (1.1) is no longer invariant under substitution (4.4) and therefore the value of the constant $C$ can not be shifted arbitrarily through rescaling of $f_{i}$ 's. The equation (4.2) still hold for the system (1.1) with integration constants $r_{0}, r_{1}$ defined as in (1.2).

As long as the condition (1.4) $(C=-\Omega / 2)$ holds and $r_{1} \neq 0, r_{0} \neq 0$ we can cast the system of equation (1.1) into (4.6) with $\kappa_{1}=\epsilon_{1} r_{0} / r_{1}$ and $\kappa_{0}=\epsilon_{0} r_{1} / r_{0}$. Thus for that case the system (1.1) is equivalent to the symmetric Painlevé $A_{3}^{(1)}$ equation (4.6) with shifted parameters $\alpha_{i} \rightarrow \bar{\alpha}_{i}$ :

$$
\bar{\alpha}_{i}=\alpha_{i}-(-1)^{[i / 2]} \frac{\epsilon_{i+1} r_{i+1}}{r_{i}}
$$

in Bäcklund relations (2.11). 
Although equation (4.8) is only valid for $r_{i} \neq 0$ it also signals what to expect when one of the integration constants goes to zero. If, for instance, $r_{1} \rightarrow 0$ then the formula (4.8) diverges for $\bar{\alpha}_{1}=\alpha_{1}+\epsilon_{0} r_{0} / r_{1}$ and $\bar{\alpha}_{3}=\alpha_{3}-\epsilon_{0} \mathrm{r}_{0} / r_{1}$ indicating breaking of $s_{1}, s_{3}$ symmetries. Interestingly, the limit $r_{1} \rightarrow 0$ can however be taken of $s_{1}$ and $s_{3}$ acting successively in the product $s_{1} s_{3}$. Observe namely that

$$
\begin{aligned}
s_{1} s_{3}\left(f_{2}\right)= & f_{2}-\frac{\bar{\alpha}_{3}}{f_{3}}+\frac{\bar{\alpha}_{1}}{f_{1}}=f_{2}-\frac{\alpha_{3}+\epsilon_{0} r_{0} / r_{1}}{-f_{1}+r_{1} z^{-C}}+ \\
& +\frac{\alpha_{1}-\epsilon_{0} r_{0} / r_{1}}{f_{1}} \underset{r_{1} \rightarrow 0}{\longrightarrow} f_{2}+\frac{\alpha_{1}+\alpha_{3}}{f_{1}}+\frac{\epsilon_{0} r_{0} z^{-C}}{f_{1}^{2}} \\
s_{1} s_{3}\left(f_{1}\right)= & f_{1}, \quad s_{1} s_{3}\left(\alpha_{0}\right)=\alpha_{0}+\alpha_{1}+\alpha_{3}, \quad s_{1} s_{3}\left(\alpha_{2}\right)=\alpha_{2}+\alpha_{1}+\alpha_{3} .
\end{aligned}
$$

Given these expressions we are able to verify that

$$
\lim _{r_{1} \rightarrow 0} s_{1} s_{3}=\pi^{2} \pi_{0} \pi_{2}
$$

with explicit expressions for symmetry generators $\pi^{2}, \pi_{0}, \pi_{2}$ that emerge below in section 4.3 as part of the $B_{2}^{(1)}$ symmetry structure obtained in the limit $r_{1} \rightarrow 0$ for an arbitrary $C$.

\section{3. $B_{2}^{(1)}$-model for $\boldsymbol{r}_{1}$ being zero}

In this case we set one of the integration constants, chosen here to be $r_{1}$, to zero. Note, that setting $r_{1}=0$ effectively leaves only one deformation parameter $\epsilon_{0}$ as $\epsilon_{1}$ appears in equation (1.1) only in the product with $r_{1}$. For $r_{1}=0$ it follows that $f_{1}+f_{3}=0$ and $\alpha_{3}=\alpha_{1}=\left(\Omega-\alpha_{0}-\alpha_{2}\right) / 2$. Consequently, equation (1.1) reduces to

$$
\begin{aligned}
& z \frac{\mathrm{d} f_{0}}{\mathrm{~d} z}=2 f_{0} f_{1} f_{2}+\left(2 \alpha_{1}+C\right) f_{0}+\alpha_{0}\left(f_{0}+f_{2}\right), \\
& z \frac{\mathrm{d} f_{1}}{\mathrm{~d} z}=f_{1}^{2}\left(f_{0}-f_{2}\right)-\left(2 \alpha_{1}+C\right) f_{1}+\epsilon_{0}\left(f_{0}+f_{2}\right), \\
& z \frac{\mathrm{d} f_{2}}{\mathrm{~d} z}=-2 f_{2} f_{0} f_{1}+\left(2 \alpha_{1}+C\right) f_{2}+\alpha_{2}\left(f_{0}+f_{2}\right) .
\end{aligned}
$$

The system (4.11) provides a symmetric representation of Painlevé III for an arbitrary parameter $C$.

Note, that a similar structure appeared earlier in [21] and this structure can be obtained from (4.11) by setting $\eta=\epsilon_{0}$ and introducing $\bar{f}_{1}=\sqrt{t} f_{1}, \quad \bar{f}_{i}=f_{i} / \sqrt{t}, \quad i=0,2$ for $C=-1 / 2, \Omega=1$.

We will now study symmetries of equations (4.11). In addition to an obvious identity automorphism: $f_{i} \rightarrow f_{i}, \alpha_{i} \rightarrow \alpha_{i}$ there also exists a sign reversal automorphism:

$f_{i} \rightarrow-f_{i}, \alpha_{i} \rightarrow \alpha_{i}$ of order 2 . A sign reversal automorphism requires a corresponding transformation of $z$ to be consistent with the second of conditions listed in (1.2). For example, for $C+\Omega=1$, the consistency requires $z \rightarrow-z$ to agree with the transformation $f_{0}, f_{2} \rightarrow$ $-f_{0},-f_{2}$.

Equations (4.11) are invariant under $\pi^{2}$ being 'the square root' of an identity automorphism defined on extended parameter space that includes $\epsilon_{0}$ :

$$
\pi^{2}: f_{1} \rightarrow-f_{1}, f_{2} \rightarrow f_{0}, f_{0} \rightarrow f_{2}, \alpha_{0} \rightarrow \alpha_{2}, \alpha_{2} \rightarrow \alpha_{0}, \epsilon_{0} \rightarrow-\epsilon_{0} .
$$

In addition, equations (4.11) are invariant under 'the square root' of a sign reversal automorphism that involves transformations of $z$ with imaginary parameters: 


$$
\rho: f_{1} \rightarrow \mathrm{i} f_{1}, f_{2} \rightarrow \mathrm{i} f_{0}, f_{0} \rightarrow \mathrm{i} f_{2}, \alpha_{0} \rightarrow \alpha_{2}, \alpha_{2} \rightarrow \alpha_{0}, z^{C+\Omega} \rightarrow \mathrm{i} z z^{C+\Omega},
$$

and $\rho\left(\epsilon_{0}\right)=\epsilon_{0}$.

Note that $\rho$ is an automorphism of order 4 and $\pi^{2}$ and $\rho$ commute

$$
\rho \pi^{2}=\pi^{2} \rho .
$$

Equation (4.11) remains invariant under $s_{0}$ and $s_{2}$ :

$$
\begin{aligned}
& s_{0}: f_{1} \rightarrow f_{1}+\frac{\alpha_{0}}{f_{0}}, f_{0} \rightarrow f_{0}, f_{2} \rightarrow f_{2}, \alpha_{0} \rightarrow-\alpha_{0}, \alpha_{2} \rightarrow \alpha_{2}, \alpha_{1} \rightarrow \alpha_{1}+\alpha_{0} \\
& s_{2}: f_{1} \rightarrow f_{1}-\frac{\alpha_{2}}{f_{2}}, f_{0} \rightarrow f_{0} f_{2} \rightarrow f_{2}, \alpha_{0} \rightarrow \alpha_{0}, \alpha_{2} \rightarrow-\alpha_{2}, \alpha_{1} \rightarrow \alpha_{1}+\alpha_{2} .
\end{aligned}
$$

For $\epsilon_{0} \neq 0$ the system (4.11) is no longer invariant under $s_{1}, s_{3}$ transformations defined in equation (2.11), which together with $s_{0}, s_{2}$ were part of $A_{3}^{(1)}$ symmetry structure of PV equation (4.1). However for $\epsilon_{0} \neq 0$ there emerge two additional symmetries given by:

$$
\begin{aligned}
\pi_{0}: f_{1} & \rightarrow-\frac{\epsilon_{0}}{f_{1}}, f_{0} \rightarrow \frac{-1}{\epsilon_{0}}\left(f_{1}^{2} f_{2}-\alpha_{2} f_{1}\right)+\left(f_{0}+f_{2}\right), f_{2} \rightarrow \frac{f_{1}}{\epsilon_{0}}\left(f_{1} f_{2}-\alpha_{2}\right), \\
\alpha_{0} & \rightarrow 2 \Omega+2 C-\alpha_{0}, \alpha_{2} \rightarrow \alpha_{2} \\
\pi_{2}: f_{1} & \rightarrow \frac{\epsilon_{0}}{f_{1}}, f_{0} \rightarrow-\frac{f_{1}}{\epsilon_{0}}\left(f_{1} f_{0}+\alpha_{0}\right), f_{2} \rightarrow \frac{1}{\epsilon_{0}}\left(f_{1}^{2} f_{0}+\alpha_{0} f_{1}\right)+\left(f_{0}+f_{2}\right), \\
\alpha_{0} & \rightarrow \alpha_{0}, \alpha_{2} \rightarrow 2 \Omega+2 C-\alpha_{2},
\end{aligned}
$$

which for $C=-\Omega / 2$ are related to $s_{1}, s_{3}$ transformations of $A_{3}^{(1)}$-model through a limiting procedure introduced in (4.10).

The Bäcklund transformations $s_{0}, s_{2}, \pi^{2}, \pi_{0}, \pi_{2}$ all square to one:

$$
s_{0}^{2}=1, s_{2}^{2}=1,\left(\pi^{2}\right)^{2}=1, \pi_{0}^{2}=1, \pi_{2}^{2}=1 .
$$

While $\rho^{4}=1$. In addition they satisfy the following fundamental Bäcklund relations:

$\pi^{2}\left(s_{0}, s_{2}, \pi_{0}, \pi_{2}\right)=\left(s_{2}, s_{0}, \pi_{2}, \pi_{0}\right) \pi^{2}, \quad \rho\left(s_{0}, s_{2}, \pi_{0}, \pi_{2}\right)=\left(s_{2}, s_{0}, \pi_{2}, \pi_{0}\right) \rho$

as well as

$$
\begin{aligned}
& \left(s_{0} s_{2}\right)^{2}=1, \quad\left(\pi_{0} \pi_{2}\right)^{2}=1, \\
& \left(\pi_{0} s_{2}\right)^{2}=1, \quad\left(\pi_{2} s_{0}\right)^{2}=1 .
\end{aligned}
$$

Relations (4.16) amount to commutativity of $s_{0}$ with $s_{2}$ and $\pi_{0}$ with $\pi_{2}$ :

$$
s_{0} s_{2}=s_{2} s_{0}, \quad \pi_{0} \pi_{2}=\pi_{2} \pi_{0},
$$

while relations (4.17) are equivalent to commutativity of $s_{0}$ with $\pi_{2}$ and $s_{2}$ with $\pi_{0}$ :

$$
s_{0} \pi_{2}=\pi_{2} s_{0}, \quad s_{2} \pi_{0}=\pi_{0} s_{2} .
$$

The last two relations transform into each other under $\pi^{2}$ conjugation. The reference [21] introduced in a setting of symmetric PIII equation a generator $s_{1}^{s}$, which in our case corresponds to $\pi^{2} \pi_{0} \pi_{2}$ for $C=-\Omega / 2=-1 / 2$. The identities $\left(s_{0} s_{1}^{s}\right)^{4}=1$ and $\left(s_{0} s_{1}^{s}\right)^{4}=1$ in reference [21] can easily be verified as direct consequences of (4.16) and (4.17).

Below, we will discuss relation of the above symmetries to the extended affine Weyl $B_{2}^{(1)}$ group. Let us inspect action of transformations $s_{2}, \pi_{0}, \pi^{2}$ : 


$$
\begin{aligned}
\left(\begin{array}{l}
\alpha_{0} \\
\alpha_{2}
\end{array}\right) \stackrel{s_{2}}{\longrightarrow}\left(\begin{array}{c}
\alpha_{0} \\
-\alpha_{2}
\end{array}\right) & \rightarrow\left(\begin{array}{l}
v_{1} \\
v_{2}
\end{array}\right) \stackrel{s_{2}}{\longrightarrow}\left(\begin{array}{c}
v_{2} \\
v_{1}
\end{array}\right), \\
\left(\begin{array}{l}
\alpha_{0} \\
\alpha_{2}
\end{array}\right) \stackrel{\pi^{2}}{\longrightarrow}\left(\begin{array}{c}
\alpha_{2} \\
\alpha_{0}
\end{array}\right) & \rightarrow\left(\begin{array}{l}
v_{1} \\
v_{2}
\end{array}\right) \stackrel{\pi^{2}}{\longrightarrow}\left(\begin{array}{c}
v_{1} \\
-v_{2}
\end{array}\right), \\
\left(\begin{array}{l}
\alpha_{0} \\
\alpha_{2}
\end{array}\right) \stackrel{\pi_{0}}{\longrightarrow}\left(\begin{array}{c}
2 \Omega+2 C-\alpha_{0} \\
\alpha_{2}
\end{array}\right) & \rightarrow\left(\begin{array}{l}
v_{1} \\
v_{2}
\end{array}\right) \stackrel{\pi_{0}}{\longrightarrow}\left(\begin{array}{l}
-1-v_{2} \\
-1-v_{1}
\end{array}\right)
\end{aligned}
$$

on $v_{1}, v_{2}$ variables:

$$
v_{1}=\frac{-1}{2(\Omega+C)}\left(\alpha_{0}+\alpha_{2}\right), \quad v_{2}=\frac{1}{2(\Omega+C)}\left(\alpha_{2}-\alpha_{0}\right),
$$

valid for $C \neq-\Omega$ (for $C=-\Omega$ the symmetry group simplifies into action of two $A_{1}^{(1)}$ groups).

One sees that actions of $\pi_{0}, s_{2}, \pi^{2}$ on parameters $\left(v_{1}, v_{2}\right)$ realize a representation of the extended affine Weyl group for the root system $B_{2}^{(1)}[7,11,18,23]$. To see the connection to the $B_{2}^{(1)}$ root lattice consider a two-dimensional vector space $\mathbf{V}$ consisting of vectors $\mathbf{v}=\mathrm{v}_{1} \mathbf{e}_{\mathbf{1}}+v_{2} \mathbf{e}_{\mathbf{2}}$, with $v_{1}, v_{2}$ being parameters of the Painlevé III equation and $\mathbf{e}_{\mathbf{1}}, \mathbf{e}_{\mathbf{2}}$ being a canonical basis of $\mathbf{V}$. Define next a symmetric bilinear form $\langle\cdot \mid \cdot\rangle$ in $\mathbf{V}$ such that $\left\langle\mathbf{e}_{i} \mid \mathbf{e}_{j}\right\rangle=\delta_{i j}$. Then according to [18] vectors

$$
\mathbf{a}_{1}=\mathbf{e}_{1}-\mathbf{e}_{2}, \quad \mathbf{a}_{2}=\mathbf{e}_{2}
$$

are the fundamental roots of the $B_{2}$ root system and

$$
\mathbf{a}_{0}=\mathbf{e}_{1}+\mathbf{e}_{2}
$$

is its highest root.

Reflections in $\mathbf{V}$ with respect to the lines $\left\langle\mathbf{a}_{i} \mid \mathbf{v}\right\rangle=0, i=1,2$ and $\left\langle\mathbf{a}_{0} \mid \mathbf{v}\right\rangle=-1$ generate transformations (4.20). Geometrically, the transformations $s_{2}, \pi^{2}$ are reflections in the hyperplane perpendicular to vectors $\mathbf{a}_{i}, i=1,2$ and the transformation $\pi_{0}$ corresponds to reflections in the hyperplane $\left\{\mathbf{v}:\left\langle\mathbf{a}_{0} \mid \mathbf{v}\right\rangle=-1\right\}$. These hyperplanes are determined by the conditions $\left\langle\mathbf{a}_{1} \mid \mathbf{v}\right\rangle=0,\left\langle\mathbf{a}_{2} \mid \mathbf{v}\right\rangle=0$ and $\left\langle\mathbf{a}_{0} \mid \mathbf{v}\right\rangle=-1$ or alternatively by $v_{1}=v_{2}, v_{2}=0$ and $v_{1}=-1-v_{2}$, respectively.

The above discussion points to $B_{2}^{(1)} \times \mathbb{Z}_{2}$ symmetry for $v_{1}, v_{2}, \epsilon_{0}$ configuration space.

It is worthwhile to point out that thanks to the fact that we extended the configuration space to include $\epsilon_{0}$ all our $B_{2}^{(1)}$ Bäcklund transformations transform $z \rightarrow z$ without a need to include a $z \rightarrow-z$ transformation as in [7, 22].

\subsection{Hamiltonian representations of PIII-PV system}

In general case with $C, r_{0}, r_{1}$ taking arbitrary values it is useful to use Hamiltonian framework to study explicit form of symmetry operations of equation (1.1).

We start defining Hamiltonian representation by introducing canonical variables $q, p$ through

$$
\begin{aligned}
& f_{1}=q, \quad f_{3}=-q+r_{1} z^{-C} \\
& f_{2}=-p, \quad f_{0}=p+r_{0} z^{C+\Omega}
\end{aligned}
$$


Then equation (1.1) can be summarized as two Hamilton equations:

$$
\begin{aligned}
z q_{z}= & q\left(q-r_{1} z^{-C}\right)\left(2 p+z^{C+\Omega}\right)-\left(\alpha_{1}+\alpha_{3}+C\right) q \\
& +\alpha_{1} r_{1} z^{-C}+\epsilon_{0} r_{0} z^{C+\Omega} \\
z p_{z}= & -p\left(p+z^{C+\Omega}\right)\left(2 q-r_{1} z^{-C}\right)+\left(\alpha_{1}+\alpha_{3}+C\right) p \\
& -\alpha_{2} r_{0} z^{C+\Omega}+\epsilon_{1} r_{1} z^{-C}
\end{aligned}
$$

obtained from the Hamiltonian:

$$
\begin{aligned}
z H_{C}= & p\left(p+r_{0} z^{C+\Omega}\right) q\left(q-r_{1} z^{-C}\right)-\left(\alpha_{1}+\alpha_{3}+C\right) p q \\
& +\left(\alpha_{1} r_{1} z^{-C}+\epsilon_{0} r_{0} z^{C+\Omega}\right) p+\left(\alpha_{2} r_{0} z^{C+\Omega}+\epsilon_{1} r_{1} z^{-C}\right) q
\end{aligned}
$$

In the following two sub-subsections of this subsection we will study symmetries of the Hamiltonian system (4.25) for two possible values of $C$ : $C=-\Omega / 2$ and $C \neq-\Omega / 2$.

4.4.1. The case of $\boldsymbol{C}=-\Omega / 2$. In this subsubsection we impose the condition (1.4) and study the second order differential equation of $q$. We will be able to show that all Hamilton equations for arbitrary $C$ such that $C=-\Omega / 2$ can be derived by simple rescaling from one particular Hamiltonian structure associated with the fixed value of $C$ (taken here to be $C=1$ / 2). This is important for evaluating a general nature of Dirac reduction process shown in section 3 .

With condition (1.4) holding the relations (1.2) become (4.3). In this context we define the canonical variables $q, p$ through

$$
q=f_{1} z^{C}, \quad p=-f_{2} z^{-C} .
$$

In this parametrization equation (1.1) becomes

$$
\begin{aligned}
& z q_{z}=q\left(q-r_{1}\right)\left(2 p+r_{0} z^{-2 C}\right)-\left(\alpha_{1}+\alpha_{3}\right) q+\alpha_{1} r_{1}+\epsilon_{0} r_{0} \\
& z p_{z}=-p\left(p+r_{0} z^{-2 C}\right)\left(2 q-r_{1}\right)+\left(\alpha_{1}+\alpha_{3}\right) p-\alpha_{2} r_{0} z^{-2 C}-\epsilon_{1} r_{1} z^{-2 C} .
\end{aligned}
$$

Equation (4.28) follows from the Hamiltonian (4.29):

$$
\begin{aligned}
z H_{C}= & p\left(p+r_{0} z^{-2 C}\right) q\left(q-r_{1}\right)-\left(\alpha_{1}+\alpha_{3}\right) p q+\left(\alpha_{1} r_{1}+\epsilon_{0} r_{0}\right) p \\
& +\left(\alpha_{2} r_{0}+\epsilon_{1} r_{1}\right) z^{-2 C} q .
\end{aligned}
$$

The canonical transformation $q \rightarrow q z^{C}, p \rightarrow p z^{-C}$ sends the Hamilton equation (4.28) into the Hamilton equation (4.25) for appropriate values of $C$.

Eliminating $p$ from the above equation (4.28) and setting $r_{0}=1, \epsilon_{1}=-1$ we obtain for $q_{z z}:$

$$
\begin{aligned}
q_{z_{c} z_{c}}= & -\frac{1}{z_{c}} q_{z_{c}}+\left(\frac{1}{2 q}+\frac{1}{2\left(q-r_{1}\right)}\right) q_{z_{c}}^{2}-\frac{\left(\alpha_{1}+\alpha_{3}+2 \alpha_{2}+2 C-2 s\right) q\left(q-r_{1}\right)}{4 C^{2} z_{c}} \\
& -\frac{\left(\alpha_{1}+\alpha_{3}\right)\left(r_{1}\left(\alpha_{3}-\alpha_{1}\right) / 2-\epsilon_{0}\right) q}{4 C^{2} z_{c}^{2}\left(q-r_{1}\right)}-\frac{\left(\alpha_{1} r_{1}+\epsilon_{0}\right)^{2}}{8 C^{2} z_{c}^{2}}\left(\frac{1}{q}+\frac{1}{q-r_{1}}\right) \\
& +\frac{1}{8 C^{2}} q\left(q-r_{1}\right)\left(2 q-r_{1}\right)
\end{aligned}
$$

where $z_{c}=z^{-2 C}$. 
Note that the term $\alpha_{1}+\alpha_{3}+2 \alpha_{2}+2 C$ can be rewritten $\alpha_{2}-\alpha_{0}$ for all the models with $C \neq 0$.

It is interesting to note that the Hamilton equation (4.28) can be rewritten as

$$
z_{c} p_{z_{c}}=-\frac{-1}{2 C} \frac{\partial\left(z H_{C=-1 / 2}\right)}{\partial q}, \quad z_{c} q_{z_{c}}=\frac{-1}{2 C} \frac{\partial\left(z H_{C=-1 / 2}\right)}{\partial q},
$$

in terms of one single $H_{C=-1 / 2}$ Hamiltonian from equation (3.4) for $r_{0}=1, \epsilon_{1}=-1$. Thus the special case of $C=-1 / 2$ carries all information about any Hamiltonian system with $C=-\Omega / 2$. It is therefore worthwhile to now consider a special example of $C=-1 / 2$ with Hamiltonian $H_{C=-1 / 2}$ giving rise to equation (3.21) when we set $r_{0}=1$ and $\epsilon_{1}=-1$. Plugging $C=-1 / 2$ into (4.30) one obtains

$$
\begin{aligned}
q_{z z}= & -\frac{1}{z} q_{z}+\left(\frac{1}{2 q}+\frac{1}{2\left(q-r_{1}\right)}\right) q_{z}^{2}-\frac{\left(\alpha_{1}+\alpha_{3}+2 \alpha_{2}-1-2 r_{1}\right) q\left(q-r_{1}\right)}{z} \\
& -\frac{\left(\alpha_{1}+\alpha_{3}\right)\left(r_{1}\left(\alpha_{3}-\alpha_{1}\right) / 2-\epsilon_{0}\right) q}{z^{2}\left(q-r_{1}\right)}-\frac{\left(\alpha_{1} r_{1}+\epsilon_{0}\right)^{2}}{2 z^{2}}\left(\frac{1}{q}+\frac{1}{q-r_{1}}\right) \\
& +\frac{1}{2} q\left(q-r_{1}\right)\left(2 q-r_{1}\right) .
\end{aligned}
$$

For $r_{1}=0$ the substitution:

$$
\tau=\sqrt{z}, \quad q=\frac{y}{\sqrt{z}}
$$

into equation (4.31) yields the Painlevé III equation:

$$
y_{\tau \tau}=\frac{y_{\tau}^{2}}{y}-\frac{y_{\tau}}{\tau}-\frac{4\left(\alpha_{2}-\alpha_{0}\right)}{\tau} y^{2}-\frac{4 \epsilon_{0}\left(\alpha_{1}+\alpha_{3}\right)}{\tau}+2 y^{3}-\frac{4 \epsilon_{0}^{2}}{y} .
$$

We note that for $r_{1} \neq 0$ the parameter $\epsilon_{0}$ can be absorbed by simple redefinitions $\alpha_{1} \rightarrow$ $\alpha_{1}-\epsilon_{0} / r_{1}, \alpha_{3} \rightarrow \alpha_{3}+\epsilon_{0} / r_{1}$, this is contrary to the situation in equation (1.5) with $C \neq$ $-\Omega / 2$, where the $\epsilon_{0}$-term could not be eliminated by redefinition of parameters. Furthermore the simple change of transformation $q \rightarrow y=1-r_{1} / q$ casts equation (4.31) in a standard PV form. This is fully consistent with an observation that for $r_{0} \neq 0, r_{1} \neq 0$ equation (1.1) describes the $A_{3}^{(1)} \mathrm{PV}$ system.

In summary, we have seen that the model for $C \neq 0$ satisfying the condition (1.4) effectively contains only one deformation parameter $r_{1}$ and the value of $r_{1}$ determines the type of the Painlevé equation described by the model with either the Painlevé III equation for $r_{1}=0$ and the PV equation for $r_{1} \neq 0$.

4.4.2. The case of $C \neq-\Omega / 2$. Since $C \neq-\Omega / 2$ we can not automatically conclude here that the model is invariant under $A_{3}^{(1)}$ extended affine Weyl group for $r_{0} \neq 0, r_{1} \neq 0$ and we have to involve the values of deformation parameters $\epsilon_{i}, i=0,1$ in the symmetry analysis.

Let us start with the simplest case of $\epsilon_{1}=0$ and $\epsilon_{0}=0$. In such case the Hamilton equation (4.25) of $H_{C}$ are invariant under transformations:

$s_{0}(q)=q+\frac{\alpha_{0}}{r_{0} z^{C+\Omega}+p}, s_{0}(p)=p, s_{0}\left(\alpha_{2 k}\right)=-(-1)^{k} \alpha_{2 k}, s_{0}\left(\alpha_{2 k+1}\right)=\alpha_{2 k+1}+\alpha_{0}$, 
$k=0,1$ as well as

$s_{2}(q)=q+\frac{\alpha_{2}}{p}, s_{2}(p)=p, s_{2}\left(\alpha_{2 k}\right)=(-1)^{k} \alpha_{2 k}, s_{2}\left(\alpha_{2 k+1}\right)=\alpha_{2 k+1}+\alpha_{2}$,

for $k=0,1$. In addition as long as $\epsilon_{0}=0, \epsilon_{1}=0$ one can also define symmetry transformations

$$
\pi(q)=-p \frac{r_{1} z^{-C}}{r_{0} z^{C+\Omega}}, \quad \pi(p)=\frac{r_{0} z^{C+\Omega}}{r_{1} z^{-C}}\left(q-r_{1} z^{-C}\right), \quad \pi\left(\alpha_{i}\right)=\alpha_{i+1}
$$

$s_{1}(p)=p-\frac{\alpha_{1}}{q}, s_{1}(q)=q, s_{1}\left(\alpha_{2 k+1}\right)=-(-1)^{k} \alpha_{2 k+1}, s_{1}\left(\alpha_{2 k}\right)=\alpha_{2 k}+\alpha_{1}$,

for $k=0,1$ and $s_{3}=\pi^{2} s_{1} \pi^{2}$. Despite its unconventional form the above automorphism $\pi$ satisfies Bäcklund relations $\pi s_{i}=s_{i+1} \pi$ and together with $s_{i}, i=0,1,2,3$ form the $A_{3}^{(1)}$ extended affine Weyl group symmetry of the Hamilton equation (4.25) as long as $\epsilon_{i}=0$, $i=0,1$. Note that due to $\epsilon_{i}=0, i=0,1$ it is in this case anyway possible to shift the value of $C$ in (1.1) to ensure that the condition (1.4) is satisfied

If we set $\epsilon_{1}=0$ and $\epsilon_{0} \neq 0$, the Hamilton equation (4.25) of $H_{C}$ are no longer invariant under $\pi, s_{1}, s_{3}$ but remain invariant under

$\pi^{2}(q)=-q+r_{1} z^{-C}, \quad \pi^{2}(p)=-p-r_{0} z^{C+\Omega}, \pi^{2}\left(\alpha_{i}\right)=\alpha_{i+2}, \quad \pi^{2}\left(\epsilon_{0}\right)=-\epsilon_{0}$

as well as $s_{0}$ and $s_{2}=\pi^{2} s_{0} \pi^{2}$. Together $s_{0}, s_{2}, \pi^{2}$ form the extended affine Weyl group $A_{1}^{(1)}$.

For $\epsilon_{0} \neq 0, \epsilon_{1}=0$ one can define birational symplectic transformations

$$
\begin{aligned}
& \bar{\pi}_{0}(q)=-\frac{\epsilon_{0}}{q}, \bar{\pi}_{0}(p)=\frac{1}{\epsilon_{0}}\left(q^{2} p+\alpha_{2} q\right) \\
& \bar{\pi}_{2}(q)=\frac{\epsilon_{0}}{q}, \bar{\pi}_{2}(p)=\frac{-1}{\epsilon_{0}}\left(q^{2}\left(p+r_{0} z^{C+\Omega}\right)+\left(\alpha_{1}+\alpha_{2}+\alpha_{3}+C\right) q+\epsilon_{0} r_{0} z^{C+\Omega}\right) .
\end{aligned}
$$

That transform the Hamiltonian $H_{C}$ from (4.26) into another polynomial in $p, q$ and thus preserve the holonomy of the system [20].

For $r_{1}=0$ (and therefore $\epsilon_{1}$ effectively eliminated from (1.1)) these transformations give rise to new symmetries:

$$
\begin{aligned}
\pi_{0}(q) & =\frac{-\epsilon_{0}}{q}, \quad \pi_{0}(p)=\frac{1}{\epsilon_{0}}\left(q^{2} p+\alpha_{2} q\right) \\
\pi_{0}\left(\alpha_{0}\right) & =2 \Omega+2 C-\alpha_{0}, \quad \pi_{0}\left(\alpha_{2}\right)=\alpha_{2} \\
\pi_{0}\left(\alpha_{1}\right) & =\alpha_{1}+\alpha_{0}-\Omega-C, \quad \pi_{0}\left(\alpha_{3}\right)=\alpha_{3}+\alpha_{0}-\Omega-C
\end{aligned}
$$

and $\pi_{2}$ such that $\pi_{2}=\pi^{2} \pi_{0} \pi^{2}$ in which we recognize $B_{2}^{(1)}$ symmetries given in (4.15) in a different basis.

4.4.3. The case of $C=0$. We now focus on the case of $C=0$ that clearly falls into a category of $C \neq-\Omega / 2$ since $\Omega$ is a constant different from zero.

All the equations and symmetry results of sections 4.4 and 4.4 .2 follow just by inserting $C=0$ into the appropriate places.

Especially, the Hamiltonian (3.3) and equation (3.10) are obtained from the Hamiltonian (4.26) and equation (4.25) by setting $C=0, r_{0}=1$ and $\epsilon_{1}=0$. Eliminating $p$ from equation (3.10) for $C=0$ yields equation (1.5): 


$$
\begin{aligned}
q_{z z}= & -\frac{1}{z} q_{z}+\left(\frac{1}{2 q}+\frac{1}{2\left(q-r_{1}\right)}\right)\left(q_{z}^{2}-\epsilon_{0}^{2}\right)-\frac{\left(\alpha r_{1}-\epsilon_{0} z \alpha_{3}\right) q}{z^{2}\left(q-r_{1}\right)} \\
& -\frac{\beta r_{1}\left(q-r_{1}\right)+\epsilon_{0} r_{1} z \alpha_{1}}{z^{2} q}-\frac{\gamma q\left(q-r_{1}\right)-\epsilon_{0}\left(1+\alpha_{1}\right)}{z} \\
& +\frac{1}{2} q\left(q-r_{1}\right)\left(2 q-r_{1}\right)
\end{aligned}
$$

with constants $\alpha, \beta, \gamma$ given by:

$$
\alpha=\frac{1}{2} \alpha_{3}^{2}, \quad \beta=-\frac{1}{2} \alpha_{1}^{2}, \quad \gamma=\alpha_{2}-\alpha_{0} .
$$

The extra parameters $r_{1}, \epsilon_{0}$ measure level of symmetry breaking.

- For $\epsilon_{0}=0, r_{1} \neq 0$ the above system is invariant under $s_{i}, i=0,1,2,3, \pi$ and (1.5) becomes the PV equation for $y=\left(q-r_{1}\right) / q$.

- For $\epsilon_{0} \neq 0, r_{1}=0$ the above system is invariant under $s_{i}, i=0,2, \pi^{2}, \pi_{0}, \pi_{2}$ and (1.5) in this limit goes to the Painlevé III equation.

- For $\epsilon_{0} \neq 0, r_{1} \neq 0$ the above Hamilton equations are only invariant under $s_{0}, \pi^{2}$ and its composite $s_{2}$. In view of the result establishing the Painlevé test for this equation (see the end of this subsection below) this equation can be viewed as a mixture of Painlevé III and Painlevé V equations.

- Finally, for the special case of $\epsilon_{0}=0, r_{1}=0$ the above system has additional symmetries and can be solved by quadratures as discussed in (4.5).

Let us now address the question whether equation (1.5) passes the Painlevé test for arbitrary values of deformation parameters $r_{1}, \epsilon_{0}$. First by inserting

$$
q(z)=a_{0}\left(z-z_{0}\right)^{\mu}
$$

and focusing on the dominant behavior near singularity on both sides of equation (1.5) we obtain

$$
\mu(\mu-1) a_{0}\left(z-z_{0}\right)^{\mu-2}=\frac{a_{0}^{2} \mu^{2}\left(z-z_{0}\right)^{2 \mu-2}}{a_{0}\left(z-z_{0}\right)^{\mu}}+a_{0}^{3}\left(z-z_{0}\right)^{3 \mu}
$$

with contributions on the right-hand side originating from the second and last term of the right-hand side of equation (1.5). This way we obtain:

$$
a_{0}^{2}=1, \quad \mu=-1
$$

consistent with the Painlevé requirement that $\mu$ is a negative integer for a movable pole with no branching. Next to check the resonance condition we plug

$$
q(z)=a_{0}\left(z-z_{0}\right)^{-1}+\eta\left(z-z_{0}\right)^{-1+r}
$$

into equation (1.5) and keep only the terms linear in $\eta$ to obtain the resonance equation for $r$ :

$$
(r+1)(r-2)=0
$$

which is identical to the resonance condition for PIII (see [8]). This resonance structure suggests that a Laurent expansion 


$$
\begin{aligned}
q(z)= & \sum_{j=0}^{\infty} a_{j}\left(z-z_{0}\right)^{j-1}=a_{0}\left(z-z_{0}\right)^{-1} \\
& +a_{1}+h\left(z-z_{0}\right)+a_{3}\left(z-z_{0}\right)^{2}+a_{4}\left(z-z_{0}\right)^{3}+\cdots
\end{aligned}
$$

expresses expansion around an arbitrary pole at $z_{0}$ where we identified $a_{2}=h$ as the single arbitrary coefficient. Direct insertion yields

$$
a_{0}^{2}=1, \quad a_{1}=\frac{1}{2 z_{0}}\left(z_{0} r_{1}+\alpha_{2}-\alpha_{0} \pm 1\right)
$$

and confirms that $a_{2}=h$ is arbitrary with all higher coefficients $a_{j}, j \geqslant 3$ uniquely determined by lower coefficients of the Laurent expansion. These observations verify successfully the Painlevé test for equation (1.5).

\subsection{Solvable model: $r_{1}=0, \epsilon_{0}=0$}

The case with both $r_{1}=0$ and $\epsilon_{0}=0$ is solvable by quadratures. With $\epsilon_{1}$ effectively eliminated due to $r_{1}=0$ and $\epsilon_{0}$ put to zero the model becomes a submodel of PV. Introducing $F=f_{1} f_{2}$ one can rewrite in such case (4.11) as

$$
\begin{aligned}
& z^{-C+1} F_{z}=f_{1}\left(-F+\alpha_{2}\right), \\
& z f_{1 z}=-2 f_{1} F+z^{-C} f_{1}^{2}+\left(C+\alpha_{0}+\alpha_{2}\right) f_{1},
\end{aligned}
$$

where we set $r_{0}=1$ (in addition to $r_{1}=0$ and $\epsilon_{0}=0$ ). One obtains from $(4.37 a)$ :

$$
f_{1}=\frac{z^{C+1} F_{z}}{\alpha_{2}-F} .
$$

Plugging this into $(4.37 b)$ yields

$$
z F_{z z}=\left(z F_{z}-F\right)_{z}=\left(-F^{2}+\left(-1+\alpha_{0}+\alpha_{2}\right) F\right)_{z}
$$

and we notice that dependence on $C$ dropped out in the above equation. After integration the above Ricatti equation can be 'linearized' by substituting:

$$
F=-z(\ln \psi)_{z}
$$

that yields the linear second order equation:

$$
z \psi_{z z}+\left(1-\alpha_{0}-\alpha_{2}\right) \psi_{z}+K_{1} \psi / z=0
$$

that appears to be a Bessel equation with solution

$\psi(z)=A_{1} \sqrt{z} J_{\sqrt{1-4 K_{1}}}\left(2 \sqrt{1-\alpha_{0}-\alpha_{2}} \sqrt{z}\right)+A_{2} \sqrt{z} Y_{\sqrt{1-4 K_{1}}}\left(2 \sqrt{1-\alpha_{0}-\alpha_{2}} \sqrt{z}\right)$

where $K_{1}$ is an integration constant [9].

\section{Discussion}

The $A_{3}^{(1)}$ PV equation is here modified by introducing a new parameter and by addition of two terms with deformation parameters. Various symmetry structures emerging in the symmetric Painlevé III-V system obtained in such way are studied in the Hamiltonian formalism and shown to depend on mutual relations between deformation parameters, integration constants and the new explicit parameter of the underlying equations. The integrable origin of the 
symmetric Painlevé III-V system is identified with the 4-boson integrable hierarchy that reduces to the $A_{4}^{(1)}$ higher Painlevé equations in a self-similarity limit. The Dirac Lagrange multiplier reduction method is here applied to second-class constraints of the $A_{4}^{(1)}$ Painlevé Hamiltonian system to obtain generic Hamiltonians of the Painlevé III-V system.

Various submodels of PIII-V model that are governed by subgroups $A_{3}^{(1)}, B_{2}^{(1)}$ and $A_{1}^{(1)}$ are contained in a hybrid ordinary differential equation that passes the Painlevé test. Due to this result this work relates to recent papers on mixing of Painlevé equations. A model that mixes the second and the third Painlevé equations and possesses Painlevé property was obtained from a mixture of $\mathrm{mKdV}$-Sine Gordon integrable models [12]. Likewise a mixture of Lund-Regge and AKNS models was to shown to reduce to the ordinary differential equation of Painlevé type [19].

The work is in progress on performing reduction of 6-boson integrable model model to Painlevé VI and other models.

\section{Acknowledgments}

Work of HA was performed when he was recipient of 2014-2015 Fulbright US Scholar Award. Support from the US-Brazil Fulbright Commission is gratefully acknowledged. AHZ and JFG were partially supported by CNPq and DVR by Fapesp and Capes.

\section{References}

[1] Adler V E 1994 Physica D 73 330-51

[2] Ablowitz M J, Ramani A and Segur H 1978 Nuovo Cimento 23333 Ablowitz M J, Ramani A and Segur H 1980 J. Math. Phys. 21715

[3] Aratyn H, Nissimov E and Pacheva S 1994 Phys. Lett. B 33182 Aratyn H, Nissimov E, Pacheva S and Zimerman A H 1995 Int. J. Mod. Phys. A 102537

[4] Aratyn H, Gomes J F and Zimerman A H 2011 J. Phys. A: Math. Theor. 44235202

[5] Aratyn H, Gomes J F and Zimerman A H 2012 Phys. Lett. A 3761966

[6] Aratyn H, Gomes J F and Zimerman A H Nonlinear and Modern Mathematical Physics: Proc. 1st Int. Workshop (AIP Conf. Proc. vol 1212) pp 146-153 (arXiv:0909.3532)

[7] Forrester P J 2010 Log-Gases and Random Matrices (LMS-34) (Princeton, NJ: Princeton University Press)

[8] Gritsuk E V and Gromak V I 2010 Differ. Equ. 46 1379-88

[9] Gromak V, Laine I and Shimomura S 2002 Math Painlevé Differential Equations in the Complex Plane (de Gruyter Stud. Math. vol 28) (Berlin: Walter de Gruyter)

[10] Hanson A, Regge T and Teitelboim C 1976 Constrained Hamiltonian Systems (Rome: Accademia Nazionale dei Lincei)

Henneaux M and Teitelboim C 1992 Quantization of Gauge Systems (Princeton, NJ: Princeton University Press)

[11] Kajiwara K, Masuda T, Noumi M, Ohta Y and Yamada Y 2001 Funkcial. Ekvac. 44 291-307

[12] Kudryashov N A 2002 J. Phys. A: Math. Gen. 35 93-9

[13] Masuda T, Ohta Y and Kajiwara K 2002 Nagoya Math. J. 168 1-25

[14] Noumi M and Yamada Y 1998 Commun. Math. Phys. 199 281-95

[15] Noumi M and Yamada Y 1998 Funkcial. Ekvac. 41 483-503

[16] Noumi M and Yamada Y 2000 Affine Weyl group symmetries in Painlevé type equations Toward the Exact WKB Analysis of Differential Equations, Linear or Non-Linear ed C J Howls et al (Kyoto: Kyoto University Press) pp 245-259

Noumi M 2002 Affine Weyl group approach to Painlevé equations Proc. ICM (Beijing) vol 3 pp 497-510

[17] Noumi M 2004 Painlevé Equations Through Symmetry (Translations of Mathematical Monographs vol 223) (Providence, RI: American Mathematical Society) 
[18] Okamoto K 1987 Funkcial. Ekvac. 30 305-32

[19] Ruy D V and de Melo G R 2014 Discontin. Nonlinearity Complex 3 161-8

Ruy D V 2015 Commun. Nonlinear Sci. Numer. Simul. 20 113-20

[20] Sasano Y and Yamada Y 2007 Symmetry and holomorphy of Painlevé type systems RIMS, Kokyuroku B2 215-25

[21] Sasano Y 2007 arXiv:0704.2391

[22] Tsuda T, Okamoto K and Sakai H 2005 Math. Ann. 331 713-38

[23] Witte N S 2004 Proc. Am. Math. Soc. 132 1649-58 